\title{
Classical analysis of time behavior of radiation fields associated with biophoton signals
}

\author{
Jeong Ryeol Choi ${ }^{\mathrm{a}, *}$, Daeyeoul Kim ${ }^{\mathrm{b}}$, Salah Menouar ${ }^{\mathrm{c}}$, Ramazan Sever ${ }^{\mathrm{d}}$ and \\ M. Sebawe Abdalla ${ }^{\mathrm{e}}$ \\ ${ }^{a}$ Department of Radiologic Technology, Daegu Health College, Daegu, Republic of Korea \\ ${ }^{\mathrm{b}}$ National Institute for Mathematical Sciences, Yuseong-daero 1689-gil, Yuseong-gu, Daejeon, \\ Republic of Korea \\ ${ }^{\mathrm{c}}$ Laboratory of Optoelectronics and Compounds, Departement of Physics, Faculty of Science, \\ University of Ferhat Abbas Setif, Setif, Algeria \\ ${ }^{\mathrm{d}}$ Department of Physics, Middle East Technical University, Ankara, Turkey \\ ${ }^{\mathrm{e}}$ Mathematics Department, College of Science, King Saud University, Riyadh, Saudi Arabia
}

\begin{abstract}
.
BACKGROUND: Propagation of photon signals in biological systems, such as neurons, accompanies the production of biophotons. The role of biophotons in a cell deserves special attention because it can be applied to diverse optical systems.

OBJECTIVE: This work has been aimed to investigate the time behavior of biophoton signals emitted from living systems in detail, by introducing a Hamiltonian that describes the process. The ratio of the energy loss during signal dissipation will also be investigated.

METHOD: To see the adiabatic properties of the biophoton signal, we introduced an adiabatic invariant of the system according to the method of its basic formulation.

RESULTS: The energy of the released biophoton dissipates over time in a somewhat intricate way when $t$ is small. However, after a sufficient long time, it dissipates in proportion $\left(1+\lambda_{0} t\right)^{2}$ to where $\lambda_{0}$ is a constant that is relevant to the degree of dissipation. We have confirmed that the energy of the biophoton signal oscillates in a particular way while it dissipates.

CONCLUSION: This research clarifies the characteristics of radiation fields associated with biophotons on the basis of Hamiltonian dynamics which describes phenomenological aspects of biophotons signals.
\end{abstract}

Keywords: Biophoton, energy dissipation, hamiltonian, damped harmonic oscillator, biological system

\section{Introduction}

The appearance of interactions between diverse atoms and molecules is affected by radiation fields generated from various sources. Theoretical properties of radiation fields have been extensively investigated during the last several decades due to their importance in a wide range of optical systems and

\footnotetext{
${ }^{*}$ Corresponding author: Jeong Ryeol Choi, Department of Radiologic Technology, Daegu Health College, Yeongsong-ro 15, Buk-gu, Daegu 41453, Republic of Korea. E-mail: choiardor@ hanmail.net.

0928-7329/16/\$35.00 (c) 2016 - IOS Press and the authors. All rights reserved

This article is published online with Open Access and distributed under the terms of the Creative Commons Attribution NonCommercial License.
} 
photonic science [1-5]. Technological applications associated with this cover laser engineering, optical displays in electronics, optical resources of quantum computation, biological sensors, and medical therapy/diagnoses. For instance, photon creations and their interference in a waveguide device have been investigated for the purpose of implementing qubits in quantum computing systems [6,7].

A useful feature of radiation fields can be acknowledged from biophoton signals that are emitted from the cells of all living things. Biophoton signals are good examples of peculiar radiation fields [8-11]. These were firstly discovered by embryologist Gurwitsch in the early days of the twentieth century [12]. Typically, the intensity of the emitted biophoton signals is about $10^{2}-10^{3}$ photons $/\left(\mathrm{cm}^{2} \mathrm{~s}\right)[13,14]$, which is extremely weak, while they have a broad spectrum of wavelengths ranging from 200 to $800 \mathrm{~nm}$. Biophotons are a kind of dynamic coherent light which promotes chemical reactions in living cells.

Biophoton signals can be possibly applied in many ways to biomedical optics, such as cancer diagnoses [15], cell growth and differentiation analysis [16], the study of neural activity of the brain [17], and hepatoma cell research [18]. The research of biophotons can also be applied to a wide range of other industrial purposes beyond medical optics. Some of such applicable fields are bio-communication [19], quantum bio-computing [20], food quality testing [21], water pollution checks [21], and the manufacturing of cosmetics [21].

Biophotons play an important role for cellular processes. Signal propagation in the neurons of a brain or other organs may occur in the form of biophoton signals generated in them [19,22-24], as well as of electrical and chemical signals. However, the mechanism for the exchange of photons by neurons as a step of neural communication has not yet been fully clarified and understood. Jaffe confirmed the transmission of luminescent signal up to $10 \mathrm{~mm}$ distance from some intertidal marine plants to Fucus zygotes, from experiments for the influence of luminescence of neighboring plants on the polarization of the direction of outgrowth for the Fucus eggs [25]. If we extend this consequence on the phenomena of intercellular level, it may be possible to conjecture the role of biophotonic activities on neural functions such as cell-to-cell communication [22]. As a possible mechanism of neural communications, Sun et al. [23] proposed a protein-protein biophoton interaction model and Craddock et al. [24] proposed a mechanism of neuronal signaling based on coherent neural oscillations. An open question in this context is how a cell distinguishes photons incoming from the neighboring cells from those originated from the environments.

The research for biophotonic quantum information transfer from sensory organs such as retina to the cerebrum may open up new perspectives to the development of photonic quantum information science in the future. Penrose and Hameroff proposed that microtubules in biological systems play the role of quantum computers that process quantum information by utilizing evanescent biophotons [26-29]. On the basis of the Brassard's teleportation theory [30], Hosna valian et al. [31] executed a simulation for neurophotonic quantum computation under the assumption that the signals in neurons of the brain can be made of biophotons as well as of electrical signals. Many unanswered questions concerning biological quantum computations have been remained until now, accompanying controversies over related mechanisms such as quantal processes of information in neuronal networks and the neural encoding for biophoton signals in brain. If quantum bio-computing technology that mimics such possible neurophotonic computation in human brain would be developed in the future, it may greatly contribute for realizing to build commercial quantum computers that have far more superior abilities in some specific tasks compared to conventional silicon-based computing.

It is known that the intensity of real biophotons inside cells and neurons is considerably higher than that which would be expected by measuring an ultraweak bioluminescence [17,32,33]. The real intensity of the biophotons within an organ is estimated to be up to two orders of magnitude higher than the 
intensity of the measured bioluminescence $[34,35]$. The positioning of relative locations of different nucleic acids both within and between cells can be executed out using entangled biophotons, providing the potential resources for quantum coherence in a biological system, needed as a basic mechanism for quantum bio-computing [20].

In fact, biophoton science has vast domains yet to be explored and the frontiers of its possible applications are still being discovered. Interestingly, biophoton signals exhibit a characteristic of nonexponential decay. Popp and Li [11] proposed a frequency stable damped harmonic oscillator model for the description of a stimulated emission of such biophoton signals. The purpose of this work is to investigate the time behavior of a biophoton signal. Starting from the Hamiltonian that describes the biophoton system, the characteristics of biophoton energy will be investigated in detail.

\section{Analysis of a biophoton signal}

Recently, the theoretical investigation of photon energy for a non-stationary radiation has been carried out by many researchers. Still the mechanism for the emission of biophotons from living cells is not exactly understood. The latest thinking is that photons are released as a result of various molecular processes relevant to energy-carrying excitons on the surface of cells. In the semiclassical framework, it was regarded that photon signals were produced through probabilistic transitions of the organic structure which emits biophoton energy, from a higher state to a lower one. However, from detailed observations concerning the energy of biophoton signals that reveal a characteristic of non-exponential decay, it is necessary to seek some alternative formulations of the mechanism instead of that based on the semiclassical framework [9].

According to the relaxation behavior of the signal, many different formulae of the Hamiltonian for describing radiation fields are allowed [36]. Among various kinds of relaxation behaviors, the one that corresponds to a frequency stable damped harmonic oscillator may be the most typically known one. If we denote the quadrature of the photon field as $q$ and its conjugate canonical variable as $p$ in this case, the Hamiltonian for describing a stimulated emission of the biophoton signal from a living system is given by [9]

$$
H(q, p, t)=\frac{p^{2}}{2 \varepsilon_{0}\left(1+\lambda_{0} t\right)^{2}}+\frac{1}{2} \varepsilon_{0}\left(1+\lambda_{0} t\right)^{2} \omega_{0}^{2} q^{2},
$$

where $\varepsilon_{0}$ is the permittivity of the medium, $\lambda_{0}$ is a constant that is responsible for the decay of the signal, and $\omega_{0}$ is the natural frequency. If we think that Eq. (1) is dependent on time, the system is non-stationary and classified as a kind of time-dependent Hamiltonian system (TDHS). Our research for biophoton signals in this work will be confined within the classical point of view, while the same research from the quantum point of view will appear later in a separate paper.

From fundamental dynamics using the Hamiltonian, we can confirm that the equation of motion for $q$ can be written as

$$
\frac{d^{2} q}{d t^{2}}+\frac{2 \lambda_{0}}{1+\lambda_{0} t} \frac{d q}{d t}+\omega_{0}^{2} q=0
$$

Here, $2 \lambda_{0} /\left(1+\lambda_{0} t\right)$ acts like a damping factor of the signal. Due to the existence of the damping factor, the biophoton signal dissipates over time. It is interesting that the damping factor depends on time in a way that it decreases as time goes by. The solution of Eq. (2) can be represented in the form

$$
q(t)=\frac{q_{0}}{1+\lambda_{0} t} \cos \left(\omega_{0} t+\delta\right)
$$


We see from this equation that the amplitude of the signal decreases over time. However, the frequency of oscillation is always the same as the natural frequency. This is the reason why the system is called a frequency stable damped harmonic oscillator. Recall that the frequency of an ordinary damped harmonic oscillator is different from the natural frequency of the system [4].

The techniques of biophoton detection from outside the cells are in general carried out through the measurement of the radiation intensity which is the radiation energy passing through a unit area per unit time [37]. The energy of a TDHS is not always the same as the Hamiltonian of the system [38,39]. For the TDHSs, the role of the Hamiltonian is limited to that of producing the classical equation of motion of the system, and in some situations, it has nothing to do with the energy of the system. In this case, the energy of the biophoton signal is given by [4] (see Appendix)

$$
U(q, p, t)=\frac{1}{\left(1+\lambda_{0} t\right)^{2}} H(q, p, t)
$$

By inserting Eq. (1) into the above equation, we have

$$
U(q, p, t)=\frac{p^{2}}{2 \varepsilon_{0}\left(1+\lambda_{0} t\right)^{4}}+\frac{1}{2} \varepsilon_{0} \omega_{0}^{2} q^{2}
$$

Using $p=\varepsilon_{0}\left(1+\lambda_{0} t\right)^{2} d q / d t$, the formula of the energy can be recast as

$$
U(t)=\frac{1}{2} \varepsilon_{0}\left(\frac{d q}{d t}\right)^{2}+\frac{1}{2} \varepsilon_{0} \omega_{0}^{2} q^{2}
$$

Now using Eq. (3) and its time derivative, we easily show that

$$
U(t)=\frac{1}{2} \varepsilon_{0} \frac{q_{0}^{2}}{\left(1+\lambda_{0} t\right)^{2}}\left(\omega_{0}^{2}+\frac{\lambda_{0}^{2}}{\left(1+\lambda_{0} t\right)^{2}} \cos ^{2}\left(\omega_{0} t+\delta\right)+\frac{\lambda_{0} \omega_{0}}{1+\lambda_{0} t} \sin \left[2\left(\omega_{0} t+\delta\right)\right]\right) .
$$

Though this is a somewhat complicated form, it is not difficult to extract the leading behavior of the energy from this. For a particular case that $t$ is sufficiently large, this equation becomes

$$
U(t) \approx \frac{1}{2} \varepsilon_{0} \frac{\omega_{0}^{2} q_{0}^{2}}{\left(1+\lambda_{0} t\right)^{2}}
$$

Thus, for a sufficiently large $t$, the energy dissipates in a way that it is proportional to the inverse of $\left(1+\lambda_{0} t\right)^{2}$.

Let us take the time average of Eq. (7). If we suppose that $\lambda_{0}$ is small, all time-dependent functions in Eq. (7) vary relatively slowly except for sine and cosine functions. For this reason, we only consider the variance of the sine and cosine functions and other variances involving $\left(1+\lambda_{0} t\right)$ will be ignored. Then, the time average of Eq. (7) is easily evaluated to be

$$
\langle U(t)\rangle_{t}=\frac{1}{2} \varepsilon_{0} \frac{q_{0}^{2}}{\left(1+\lambda_{0} t\right)^{2}}\left(\frac{\lambda_{0}^{2}}{2\left(1+\lambda_{0} t\right)^{2}}+\omega_{0}^{2}\right) .
$$

The first term in the above equation dissipates rapidly and the second term survives in the long run, leading to the same result as that of Eq. (8). 
The time evolution of energy of the biophoton signal can also be estimated from another way that is based on the adiabatic invariant method. When some parameters of a slowly varying dynamical system undergo adiabatic change, a physical quantity that nearly does not vary with time is called an adiabatic invariant. The introduction of an adiabatic invariant is useful for investigating characteristics of timedependence of nonstationary Hamiltonian systems $[40,41]$. The adiabatic invariant $I(t)$ of the system can be obtained from the integration

$$
I(t)=\oint p d q
$$

To evaluate this quantity, let us consider the elliptic equation in phase space, which is obtained from Eq. (5) in the form

$$
\frac{p^{2}}{A_{p}^{2}}+\frac{q^{2}}{A_{q}^{2}}=1,
$$

where $A_{p}$ and $A_{q}$ are two exes that are given by

$$
\begin{aligned}
& A_{p}=\sqrt{2 \varepsilon_{0} \bar{U}(q, p, t)}\left(1+\lambda_{0} t\right)^{2}, \\
& A_{q}=\frac{1}{\omega_{0}} \sqrt{\frac{2 \bar{U}(q, p, t)}{\varepsilon_{0}}}
\end{aligned}
$$

and $\bar{U}$ is the energy of the photon signal which is in fact the same as $U$ in Eq. (5). Here, we inserted an upper bar in the expression of $\bar{U}$ in order to distinguish this energy based on the adiabatic invariant from the energy that was previously obtained from the ordinary way. From the phase orbit of the system, which is elliptic, we can derive the adiabatic invariant in the form

$$
I(t)=\frac{2 \pi \bar{U}(t)\left(1+\lambda_{0} t\right)^{2}}{\omega_{0}}
$$

According to the fundamental properties of adiabatic invariants, it is possible to put [41]

$$
I(t)=I(0)
$$

The substitution of Eq. (14) into this identity yields

$$
\bar{U}(t)=\frac{1}{\left(1+\lambda_{0} t\right)^{2}} \bar{U}(0) .
$$

As you can see, the time behavior of the energy expressed in this form is the same as that in Eq. (8). From a simplistic comparison of Eq. (16) with Eq. (8), we confirm that $\bar{U}(0)=\varepsilon_{0} \omega_{0}^{2} q_{0}^{2} / 2$. Although Eq. (16) is somewhat rough while the energy expression given in Eq. (7) is exact, the leading behavior of the energy dissipation can be obtained from Eq. (16). The graphical comparison of the time behavior of differently formulated energies given in Eqs (7) and (16) is shown in Fig. 1. From this figure, we see that the exact energy [Eq. (7)] not only decreases but also fluctuates as time goes by. The Hamiltonian of the system also oscillates, but its envelope does not decrease over time. 


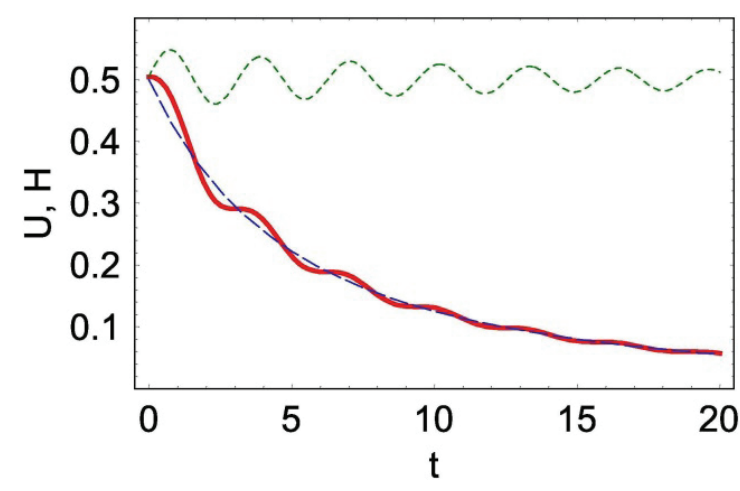

Fig. 1. Time behavior of biophoton energy given in Eq. (7) [thick line] and Eq. (16) [long dashed line]. A reference line which is the short dashed line is the time behavior of the Hamiltonian. We used $q_{0}=1, \lambda_{0}=0.1, \omega_{0}=1, \varepsilon_{0}=$ 1 , and $\delta=0$.

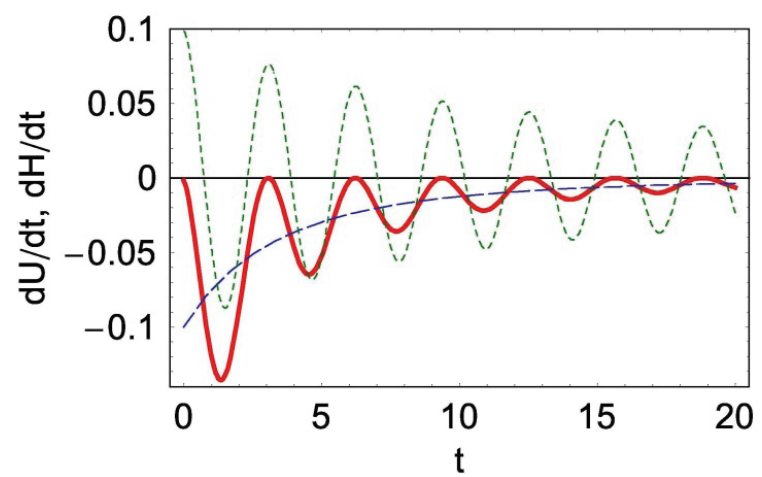

Fig. 2. Time derivative of biophoton energy given in Eq. (18) [thick line] and Eq. (19) [long dashed line]. A reference line which is the short dashed line is the time derivative of the Hamiltonian given in Eq. (17). We used $q_{0}=1, \lambda_{0}=0.1$, $\omega_{0}=1, \varepsilon_{0}=1$, and $\delta=0$.

We can estimate the ratio of the decrease of any physical quantity from its time derivative. The time derivative of the Hamiltonian and energies are given by

$$
\begin{aligned}
\frac{d H(t)}{d t}= & \frac{\varepsilon_{0} \lambda_{0} q_{0}^{2}}{2\left(1+\lambda_{0} t\right)^{3}}\left\{\left[2 \omega_{0}^{2}+4 \lambda_{0} \omega_{0}^{2} t+\lambda_{0}^{2}\left(2 \omega_{0}^{2} t^{2}-1\right)\right] \cos \left[2\left(\omega_{0} t+\delta\right)\right]\right. \\
& \left.-\lambda_{0}\left\{\lambda_{0}+2 \omega_{0}\left(1+\lambda_{0} t\right) \sin \left[2\left(\omega_{0} t+\delta\right)\right]\right\}\right\} \\
\frac{d U(t)}{d t}= & -\frac{2 \varepsilon_{0} \lambda_{0} q_{0}^{2}}{\left(1+\lambda_{0} t\right)^{5}}\left[\lambda_{0} \cos \left(\omega_{0} t+\delta\right)+\omega_{0}\left(1+\lambda_{0} t\right) \sin \left(\omega_{0} t+\delta\right)\right]^{2} \\
\frac{d \bar{U}(t)}{d t}= & -\frac{\varepsilon_{0} \lambda_{0} \omega_{0}^{2} q_{0}^{2}}{\left(1+\lambda_{0} t\right)^{3}}
\end{aligned}
$$

From Fig. 2 which is the illustration of these equations, more detailed analyses of energy loss are possible. The exact energy $U$ dissipates rapidly near the vicinity of center where $q=0$ and the energy does not dissipate at the turning point in the oscillation. On the other hand, $\bar{U}$ dissipates in a monotonic way. The Hamiltonian becomes large and small in turn and this fluctuation gradually declines as time passes.

\section{Conclusion}

The energy of a biophoton system is obtained from Hamiltonian dynamics relevant to the corresponding biophoton signal. The energy is different from the Hamiltonian of the system because the Hamiltonian is explicitly dependent on time. Both the energy and Hamiltonian oscillate with time. The envelope of oscillation of the energy dissipates with time while that of the Hamiltonian does not. Of course, the energy becomes the same as the Hamiltonian in case that the time dependence of the Hamiltonian disappears. The dissipated radiation energy converts to heat. From the analysis of biophoton signals, we can confirm that there is a non-exponential decay of the energy of photons. Such decaying property of the energy follows Eq. (7) which is a somewhat complicated form. Biophotons are the only objects that exhibit such relaxation behavior over time as far as we know. For a sufficiently large $t$, the energy decreases in a way that it is proportional to the inverse of $\left(1+\lambda_{0} t\right)^{2}$. For experimental results and data 
which support this theoretical consequence, you can refer to Refs. [8,9,36,42]. The analysis of photon energy based on the adiabatic invariant also gives the same results.

There is potential applicability of biophoton signals, emitting from cells and tissues of human beings and other biological systems, in many fields of technologies and scientific areas, as mentioned previously. In spite of extensive experimental literature concerning the emission of biophoton signals and their phenomenological features, theoretical aspects for this novel phenomenon are poorly understood currently [43]. At times, the lack of a complete and clear theory necessary for manipulating the experimental data leads to controversy. Most of the important consequences of experimental analyses associated with biophoton signals require a strong theoretical background in order to explain them on a general basis. This research provides a theoretical background for understanding the time behavior of biophoton signals. The theoretical concepts of biophoton systems are necessary for learning something new about the biophotonic emissions and the characteristics of emitted photons.

As a final remark, wave propagation in the neurons of a biological system takes place via the production of biophotons. It is worth paying special attention to the role of biophotons in cells due to their wide applicability even beyond the scope of biological science. Especially, it is expected that the characteristic of biophotons could be applied in implementing quantum information science such as quantum computing $[20,31]$. This paper clarifies the time behavior of biophoton signals on the basis of Hamiltonian dynamics which describes phenomenological aspects of biophotons.

\section{Acknowledgements}

This research was supported by the Basic Science Research Program of the year 2015 through the National Research Foundation of Korea (NRF) funded by the Ministry of Education (Grant No.: NRF2013R1A1A2062907). M.S. Abdalla extends his appreciation to the Deanship of Scientific Research at KSU for funding the work through the research group project No. PRG/1436/22.

\section{References}

[1] Louisell WH. Quantum Statistical Properties of Radiation. John Wiley and Sons; New York, 1973.

[2] Kalluri DK. Electromagnetics of Time Varying Complex Media. 2nd ed. CRC Press; Boca Raton, 2010.

[3] Choi JR. Thermal radiation fields in time-dependent linear media at finite temperature. Laser Phys Lett. 2013; 10: 105202.

[4] Choi JR. Invariant operator theory for the single-photon energy in time-varying media. Chin Phys. B. 2010; 19: 010306.

[5] Choi JR. Coherent and squeezed states of light in linear media with time-dependent parameters by Lewis-Riesenfeld invariant operator method. J Phys B: At Mol Opt Phys. 2006; 39: 669-684.

[6] Crespi A, Ramponi R, Osellame R, Sansoni L, Bongioanni I, Sciarrino F, Vallone G, Mataloni P. Integrated photonic quantum gates for polarization qubits. Nat Commun. 2011; 2: 566.

[7] Politi A, Matthews JCF, O'Brien JL. Shor's quantum factoring algorithm on a photonic chip. Science. 2009; $325: 1221$.

[8] Bajpai RP. Squeezed state description of spectral decompositions of a biophoton signal. Phys Lett A. 2005; 337: 265-273.

[9] Bajpai RP. Biophoton emission in a squeezed state from a sample of Parmelia tinctorum. Phys Lett A. 2004; 322 : 131136.

[10] Popp FA, Chang JJ, Herzog A, Yan Z, Yan Y. Evidence of non-classical (squeezed) light in biological systems. Phys Lett A. 2002; 293: 98-102.

[11] Popp FA, Li KH. Hyperbolic relaxation as a sufficient condition of a fully coherent ergodic field. Int J Theor Phys. 1993; 32: $1573-1583$.

[12] Gurwitsch A. Über Ursachen der Zellteilung. Arch Entw Mech Org. 1922; 51: 383-415.

[13] Devaraj B, Usa M, Inaba H. Biophotons: ultraweak light emission from living systems. Curr Opin Solid State Mat Sci. 1997; 2: 188-193. 
[14] Radotic K, Radenovic C, Jeremic M. Spontaneous ultraweak bioluminescence in plants: origin, mechanisms and properties. Gen. Physiol. Biophys. 1998; 17: 289-308.

[15] Hossu M, Ma L, Zou X, Chen W. Enhancement of biophoton emission of prostate cancer cells by Ag nanoparticles. Cancer Nanotechnol. 2013; 4: 21-26.

[16] Popp FA. Properties of biophotons and their theoretical implications. Indian J Exp Biol. 2003; 41: 391-402.

[17] Rahnama M, Bokkon I, Tuszynski J, Cifra M, Sardar P, Salari V. Emission of biophotons and neural activity of the brain. arXiv:1012.3371 [physics. bio-ph], 2011.

[18] van Wijk R, van Aken H. Light-induced photon emission by rat hepatocytes and hepatoma cells. Cell Biophys. 1991; 18 : $15-29$.

[19] van Wijk R. Bio-photons and bio-communication. J Sci Explor. 2001; 15: 183-197.

[20] Mihelic FM. A theoretical mechanism of Szilard engine function in nucleic acids and the implications for quantum coherence in biological systems. AIP Conf Proc. 2010; 1316: 287-290.

[21] Jung H-H, Yang J-M, Woo W-M, Choi C, Yang J-S, Soh K-S. Year-long biophoton measurements normalized frequency count analysis and seasonal dependency. J Photochem Photobiol B: Biol. 2005; 78: 149-154.

[22] Prasad A, Rossi C, Lamponi S, Pospisil P, Foletti A. New perspective in cell communication: potential role of ultra-weak photon emission. J Photochem Photobiol B: Biol. 2014; 139: 47-53.

[23] Sun Y, Wang C, Dai J. Biophotons as neural communication signals demonstrated by in situ biophoton autography. Photochem. Photobiol. Sci. 2010; 9: 315-322.

[24] Craddock TJA, Priel A, Tuszynski JA. Keeping time: Could quantum beating in microtubules be the basis for the neural synchrony related to consciousness? J Integr Neurosci. 2014; 13: 293-311.

[25] Jaffe LF. Marine plants may polarize remote Fucus eggs via luminescence. Luminescence. 2005; 20: 414-418.

[26] Penrose R. Shadows of the Mind: A Search for the Missing Science of Consciousness. Oxford University Press; Oxford, 1994;

[27] Hameroff SR, Penrose R. Conscious events as orchestrated space-time selections. J Conscious Stud. 1996; 3: 36-53.

[28] Hameroff SR. Quantum computation in brain microtubules. The Penrose-Hameroff' Orch OR model of consciousness. Philos Trans R Soc Lond A. 1998; 356: 1869-1896.

[29] Hameroff SR. The brain is both neurocomputer and quantum computer. Cognitive Sci. 2007; 31: 1035-1045.

[30] Brassard G. Teleportation as a quantum computation. Physica D. 1998; 120: 43-47.

[31] Hosna V, Bassereh H, Barkhordari A, Salari V. A simulation for neurophotonic quantum computation in visual pathways. arXiv:1412.7551v1 [q-bio. OT], 2014.

[32] Thar R, Kühl M. Propagation of electromagnetic radiation in mitochondria? J Theor Biol. 2004; 230: 261-270.

[33] Bókkon I, Salari V, Tuszynski J, Antal I. Estimation of the number of biophotons involved in the visual perception of a single object image: Biophoton intensity can be considerably higher inside cells than outside. J Photochem Photobiol B: Biol. 2010; 100: 160-166.

[34] Chwirot BW. Ultraweak luminescence studies of microsporogenesis in Larch. in Recent Advances in Biophoton Research and Its Applications. Popp FA, Li KH, Gu Q, eds. World Scientific; Singapore, 1992; pp. 259-285.

[35] Slawinski J. Luminescence research and its relation to ultraweak cell radiation. Experientia. 1988; 44: 559-571.

[36] Bajpai RP, Kumar S, Sivadasan VA. Biophoton emission in the evolution of a squeezed state of a frequency stable damped oscillator. Appl Math Comput. 1998; 93: 277-288.

[37] Blake T, Dotta BT, Buckner CA, Cameron D, Lafrenie RM, Persinger MA. Biophoton emissions from cell cultures: biochemical evidence for the plasma membrane as the primary source. Gen Physiol Biophys. 2011; 30: 301-309.

[38] Marchiolli MA, Mizrahi SS. Dissipative mass-accreting quantum oscillator. J Phys A: Math Gen. 1997; 30: $2619-2635$.

[39] Choi JR. The effects of nonextensivity on quantum dissipation. Sci Rep. 2014; 4: 3911.

[40] Goldstein H. Classical Mechanics. 2nd ed. Addison Wesley; New York, 1980, Sec. 7 of Chap. 11.

[41] Goh JG. Mechanics [in Korean]. Cheong Mun Gak; Seoul, 1998.

[42] Popp FA. Biophotons-background, experimental results, theoretical approach and applications. Res Adv Photochem Photobiol. 2000; 1: 31-41.

[43] Alvermann M, Srivastava YN. Biological electric fields and rate equations for biophotons. arXiv:1407.4689v1 [physics. gen-ph], 2014.

\section{Appendix}

In this Appendix, we show how to derive the energy expression of the biophoton signal, given in Eq. (4). In general, the energy of an arbitrary time-dependent optical wave, described by permittivity 
$\varepsilon(t)$ and conductivity $\sigma(t)$, has the form [4]

$$
U(q, p, t)=e^{-\Lambda(t)} H(q, p, t)
$$

where $\Lambda(t)$ is a time function that is represented as

$$
\Lambda(t)=\int_{0}^{t} \frac{\sigma\left(t^{\prime}\right)+d \varepsilon\left(t^{\prime}\right) / d t^{\prime}}{\varepsilon\left(t^{\prime}\right)} d t^{\prime} .
$$

For the case of frequency stable damped harmonic oscillator description of the biophoton signal, the parameters are given by [4]

$$
\varepsilon(t)=\varepsilon_{0}, \quad \sigma(t)=\frac{2 \varepsilon_{0} \lambda_{0}}{1+\lambda_{0} t}
$$

By executing the integration in Eq. (21) after inserting these quantities, we have

$$
\Lambda(t)=2 \ln \left(1+\lambda_{0} t\right) .
$$

Thus the substitution of Eq. (23) in Eq. (20) leads to Eq. (4) in the text. 